\title{
Hepatic toxicity during regorafenib treatment in patients with metastatic gastrointestinal stromal tumors
}

\author{
PHILIPP IVANYI $^{1 *}$, HENDRIK EGGERS $^{1 *}$, MAREIKE HORNIG $^{1}$, BERND KASPER $^{2}$, KLAUS HEISSNER $^{3}$, \\ HANS-GEORG KOPP $^{3}$, MARTHA KIRSTEIN ${ }^{4,5}$, ARNOLD GANSER ${ }^{1}$ and VIKTOR GRÜNWALD ${ }^{1,6}$ \\ ${ }^{1}$ Department of Hematology, Hemostasis, Oncology and Stem Cell Transplantation, \\ Hannover Medical School, D-30625 Hannover; ${ }^{2}$ Interdisciplinary Tumor Center Mannheim, \\ Mannheim University Medical Center, University of Heidelberg, D-68167 Mannheim; ${ }^{3}$ Department of Oncology, \\ Hematology, Clinical Immunology, Rheumatology and Pneumology, University Hospital and Faculty of Medicine Tübingen, \\ D-72076 Tübingen; ${ }^{4}$ Department of Gastroenterology, Hepatology and Endocrinology, Hannover Medical School, \\ D-30625 Hannover; ${ }^{5}$ First Department of Medicine-Internal Medicine, University Medical Center Schleswig-Holstein, \\ D-23538 Lübeck; ${ }^{6}$ Department of Interdisciplinary GU Oncology, West-German Cancer Center Essen, \\ University Hospital Essen, D-45147 Essen, Germany
}

Received March 4, 2020; Accepted July 31, 2020

DOI: $10.3892 / \mathrm{mco} .2020 .2143$

\begin{abstract}
Regorafenib is a multi-target tyrosine kinase inhibitor that has been approved for the treatment of metastatic colorectal cancer, advanced hepatocellular carcinoma, and metastatic gastrointestinal stromal tumors (GIST). Severe hepatobiliary toxicity has been reported in patients with colorectal cancer treated with regorafenib, but not in those with GIST. Therefore, the aim of the present study was to investigate the incidence and clinical course of regorafenib-associated hepatic toxicity (HT) in patients with GIST in a real-world setting. Patients with metastatic GIST treated with regorafenib between September 2012 and May 2014 at three German tertiary care centers were followed up until August 2017. Patient records were retrospectively analyzed and descriptive statistics were employed. HT was defined as alterations in the serum values of aspartate aminotransferase, alanine aminotransferase, $\gamma$-glutamyltransferase, alkaline phosphatase and bilirubin (according to the Common Terminology Criteria for Adverse Events, version 4.0), and/or corresponding clinical signs. The time to clinical progression and the overall survival were calculated by Kaplan-Meier curves. Overall, 21 patients were treated with regorafenib and $5(23.5 \%)$ of those heavily
\end{abstract}

Correspondence to: $\mathrm{Dr}$ Hendrik Eggers, Department of Hematology, Hemostasis, Oncology and Stem Cell Transplantation, Hannover Medical School, 1 Carl-Neuberg Street, D-30625 Hannover, Germany

E-mail: eggers.hendrik@mh-hannover.de

*Contributed equally

Key words: gastrointestinal stromal tumor, tyrosine kinase inhibitor, regorafenib, hepatic toxicity, retrospective, real-world data pretreated patients suffered from severe HT during regorafenib treatment. In $4(80 \%)$ of these cases, regorafenib treatment was continued, optimizing individual treatment benefit. Clinical monitoring and adequate therapy management are crucial for ensuring continuation of regorafenib treatment in order to achieve an optimal clinical outcome.

\section{Introduction}

Regorafenib is an oral a multi-target tyrosine kinase inhibitor blocking several protein kinases and targeting tumor angiogenesis, as well as the oncogenic kinases KIT, RET and B-RAF (1). The antitumor effect of regorafenib has been demonstrated in colorectal cancer, gastrointestinal stromal tumors (GIST) and hepatocellular cancer (2-4). Since imatinib was one of the first tyrosine kinase inhibitors to be approved for the treatment of GIST in 2002, the therapeutic landscape has changed markedly. The efficacy of tyrosine kinase inhibitors has been demonstrated for a number of other diseases, but several common side effects have also been reported, including hypertension, palmar-plantar erythrodysesthesia syndrome, diarrhea and fatigue (5).

Hepatic toxicity (HT) has been observed in patients under treatment with various tyrosine kinase inhibitors. The incidence of severe HT was estimated to be $5 \%$ in a systematic review of patients treated with various tyrosine kinase inhibitors $(3,6-9)$. In a pivotal phase III trial of regorafenib in patients with GIST $(n=132)$, apart from 1 case of drug-related fatal hepatic failure, no HT or biliary toxicity were reported (2); however, our data indicate that almost one-quarter of patients treated with regorafenib experience laboratory and/or clinical signs of HT (2).

The incidence of HT among GIST patients treated with regorafenib may be underreported. The aim of the present study was to conduct a retrospective analysis of a real-world cohort, treated at three German tertiary hospitals, in order to 
investigate the incidence of HT among GIST patients treated with regorafenib, as timely management is crucial for allowing continuation of regorafenib treatment with a durable palliative result, while severe HT may require treatment discontinuation and compromise the outcome of heavily pretreated patients.

\section{Patients and methods}

Patients and ethics statement. The present study included patients with GIST who were treated with regorafenib between September 2012 and May 2014 at three German University Hospitals (Tübingen, Heidelberg and Hannover). The last follow-up was documented in August 2017. Patient data were anonymized and assessed retrospectively. All analyses were performed in concordance with the recommendations of the local ethics committees, and after obtaining approval from the Ethics Committee of Hannover Medical School (Hannover, Germany, reference no. 3167-2016). The study protocol conformed to the principles outlined in the latest amendment of the Declaration of Helsinki (10).

Regorafenib treatment. Regorafenib was administered after obtaining written informed consent from the patients. Dosing was adjusted according to investigator's judgement following the product information. All patients received computed tomography or magnetic resonance imaging examinations at baseline and subsequently every 3 months, according to local standards. Clinical chemistry and blood count measurements were performed according to local standards.

Definition of HT. HT was defined as any alteration in the serum values of aspartate aminotransferase (AST), alanine aminotransferase (ALT), $\gamma$-glutamyltransferase $(\gamma$-GT), alkaline phosphatase (AP) and bilirubin, corresponding to an adverse event of grade $\geq 3$ according to the Common Terminology Criteria of Adverse Events (CTCAE), version 4.0, and/or by corresponding clinical signs (e.g., icterus, pruritus and exanthema) (11). The values were documented at the start of therapy, at maximum peak and at the end of therapy, or last value recorded (resolution). HT was considered as regorafenib-associated only in patients without evidence of hepatic tumor progression and no other plausible cause.

The institutional cut-off for pathological values was applied, and CTCAE grading was performed by the multiplication of the upper limit of normal.

Statistical analysis. Statistical analysis was performed using SPSS 24.0 (IBM Corp.). Descriptive patient and treatment characteristics were evaluated. Clinical progression-free survival (cPFS) was defined as the time from initiation of regorafenib treatment until the first evidence of either clinical or radiological progression, and it was calculated by Kaplan-Meier curves. Overall survival (OS) was defined as the time from initiation of regorafenib treatment until death from any cause, and it was calculated using Kaplan-Meier curves.

\section{Results}

Patient characteristics. A total of 21 patients treated with regorafenib for GIST were identified within the observation period.
Table I. Baseline patient and tumor characteristics at diagnosis.

\begin{tabular}{lc}
\hline Baseline parameters & No. $(\%)^{\mathrm{a}}$ \\
\hline Patients & $21(100.0)$ \\
Median age at diagnosis, years (range) & $67.2(31.4-87)$ \\
Sex & \\
Male & $16(76.2)$ \\
Female & $5(23.8)$ \\
Primary GIST location & \\
Esophagus & $3(14.3)$ \\
Stomach & $6(28.6)$ \\
Small intestine & $8(38.1)$ \\
Colon & $2(9.5)$ \\
Peritoneum & $2(9.5)$ \\
Median time to metastasis, months (range) & $0(0-64)$ \\
Synchronous metastasis at initial diagnosis & $12(57.1)$ \\
of GIST & \\
Synchronous liver metastasis at initial & $7(33.3)$ \\
diagnosis of GIST & \\
Primary molecular pathology & \\
c-KIT exon 11 mutation & $8(38.1)$ \\
c-KIT exon 9 mutation & \\
c-KIT wild-type & \\
Not available & \\
\hline
\end{tabular}

${ }^{\mathrm{a} U n l e s s}$ otherwise indicated. GIST, gastrointestinal stromal tumors.

The median follow-up was 14.8 months (range, 2-42 months) and the median age at diagnosis was 67.2 years (range, 31-87 years). The primary origin of GIST was mostly in the small intestine $(n=8,38.1 \%)$, followed by the stomach $(n=6$, $28.6 \%$ ). Synchronous metastases at initial diagnosis were present in 12 patients $(57.1 \%)$. A total of 7 patients $(33.3 \%)$ suffered from liver metastases at initial diagnosis, while overall hepatic metastases developed in 19 patients (90.5\%) (Table I). The patterns of metastatic spread are shown in supplementary Table SI.

Treatment. Patients received 3-8 therapeutic lines prior to the initiation of regorafenib, while the median duration of regorafenib treatment was 5.15 months (range, 2-20 months). Dose reduction of regorafenib was necessary in 15 patients $(71.4 \%)$. The subsequent therapeutic lines ranged from 0 to 5 (Table II).

Incidence of HT. A total of 5 patients with clinical and/or laboratory HT were identified (23.5\%). The median time to HT was 1.7 months (range, 0.9-11.2 months). Of these 5 patients, 1 exhibited liver progression of GIST at time of HT, while the remaining 4 patients were able to continue regorafenib treatment without reoccurrence of HT. Only 1 patient discontinued treatment with regorafenib permanently due to severe toxicities other than HT (Table III).

Laboratory changes in patients with HT. The levels of AST, ALT, $\gamma$-GT, AP and bilirubin were elevated in patients with 
A

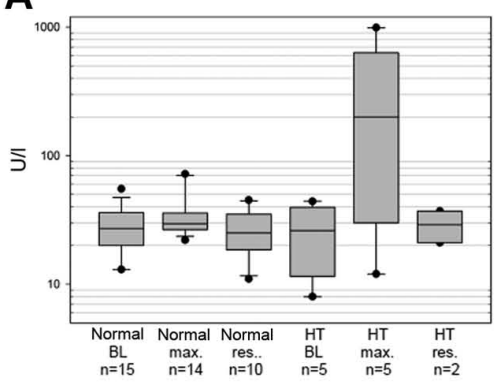

D

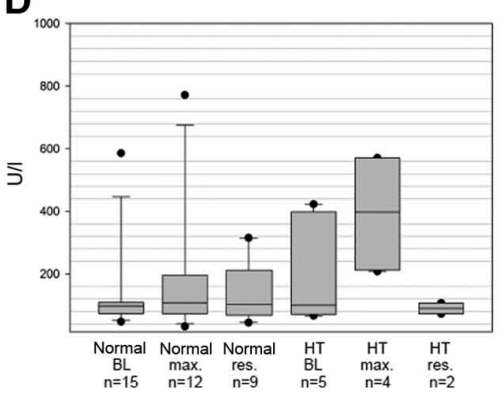

B

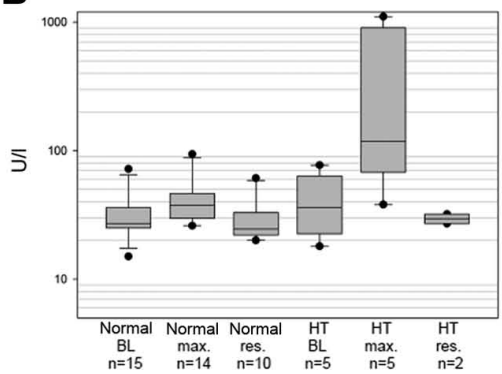

E

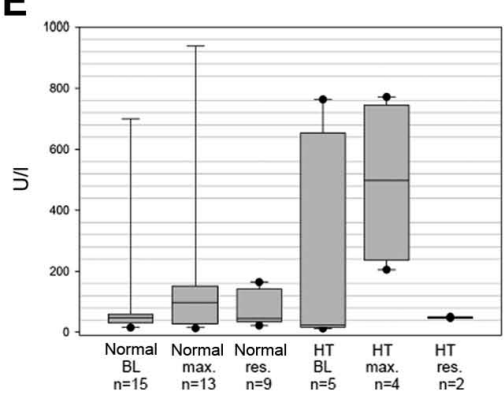

C

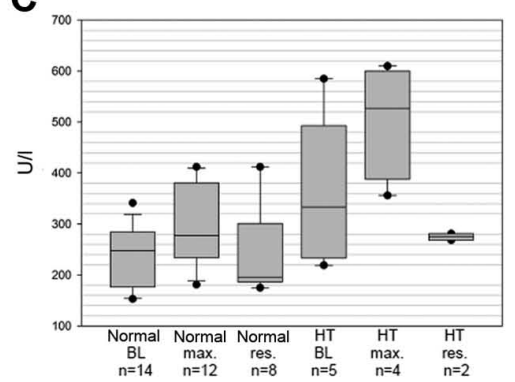

F

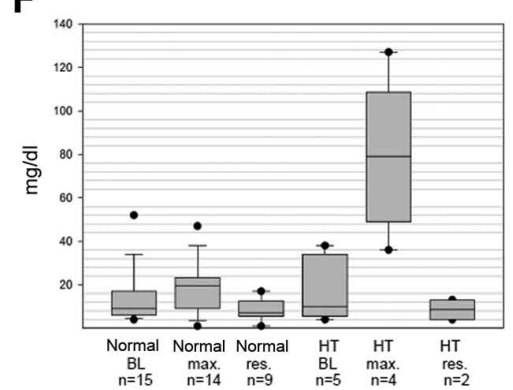

Figure 1. Box plot displaying laboratory values of (A) ALT, (B) AST, (C) LDH, (D) AP, (E) $\gamma$-GT and (F) bilirubin. $\gamma$-GT was elevated and grade $>3$ at BL for 2 patients with HT, of whom 1 patient exhibited a significant increase in AST and ALT from normal to grade 3 and 4 values, without further alteration of $\gamma$-GT; the other patient exhibited a minor increase in AST, ALT, AP and $\gamma$-GT levels. Median, interquartile range, 95\% confidence interval, maximum and minimum levels measured at BL, levels at maximum elevation (max.) and resolution of toxicity (res.) are shown for patients with HT and those without HT (normal). HT, hepatic toxicity; ALT, alanine aminotransferase; AST, aspartate aminotransferase; LDH, lactate dehydrogenase; AP, alkaline phosphatase; $\gamma$-GT, $\gamma$-glutamyltransferase; BL, baseline.

Table II. Treatment parameters of 21 patients treated with regorafenib.

Treatment parameters

No. $(\%)^{\mathrm{a}}$

Median age at regorafenib initiation, years (range) 70 (35-86)

Median number of therapeutic lines before

$4(3-8)$

regorafenib (range)

Median duration of regorafenib treatment, months 5.15 (2-20)

(range)

Regorafenib dose reduction (due to any reason)

$15(71.4)$

Reason for discontinuation of regorafenib

Disease progression

$15(71.4)$

Toxicity other than HT

Death

Not evaluable

Median number of systemic treatments after regorafenib failure (range)

$1(0-5)$

${ }^{\mathrm{a}}$ Unless otherwise indicated. HT, hepatic toxicity.

HT (Fig. 1A-F). The median maximum level of ALT in patients with HT was $200 \mathrm{U} / 1$ (range, 12-992 U/1) compared with $30 \mathrm{U} / 1$ (range, 22-72 U/l) in non-HT patients; AST, HT $118 \mathrm{U} / 1$ (range, 38-1,104 U/1) vs. non-HT 38 U/1 (range, 26-94 U/1); AP, HT 398 U/1 (range, 208-571 U/l) vs. non-HT 108 U/1 (range, 32-772 U/1); $\gamma$-GT, HT 499 U/l (range, 205-771 U/l) vs. non-HT $98 \mathrm{U} / 1$ (range, 13-1,400 U/l); bilirubin, HT $79 \mu \mathrm{mol} / 1$ (range, 36-127 $\mu \mathrm{mol} / \mathrm{l}$ ) vs. non-HT $20 \mu \mathrm{mol} / \mathrm{l}$ (range, 1-47 $\mu \mathrm{mol} / \mathrm{l}$ );
Table III. Characteristics of HT in patients treated with regorafenib.

\begin{tabular}{ll} 
Hepatic toxicity & No. $(\%)^{\mathrm{a}}$ \\
\hline $\begin{array}{l}\text { Patients with liver metastasis prior to } \\
\text { regorafenib treatment }\end{array}$ & $19(90.5)$ \\
Number of patients with HT & $5(23.5)$ \\
HT laboratory value alterations & $4(80.0)$ \\
Clinical signs only of HT & $1(20.0)$ \\
Median time to HT, months (range) & $1.7(0.9-11.2)$ \\
HT patients on hepatotoxic comedication & $3(14.3)$ \\
Liver progression at the time of HT & $1(4.8)$ \\
Regorafenib continuation after HT & $4(19.0)$ \\
Reoccurrence of HT & $0(0.0)$ \\
\hline
\end{tabular}

${ }^{a}$ Unless otherwise indicated. ${ }^{b}$ Liver progression defined as new and/or larger tumor lesions within the liver on imaging. HT, hepatic toxicity.

and lactate dehydrogenase (LDH), HT 527 U/l (range, 356-610 U/l) vs. non-HT 278 U/l (range, 181-412 U/l). The last available values, or those measured at the end of treatment, did not differ significantly from baseline levels. The median ALT at resolution in patients with HT was $29 \mathrm{U} / 1$ (range, 21-37 U/1), the AST was $30 \mathrm{U} / 1$ (range, 27-32 U/1), the AP was $90 \mathrm{U} / 1$ (range, 72-107 U/1), the $\gamma$-GT was $48 \mathrm{U} / 1$ (range, 46-50 U/1), the bilirubin was $9 \mu \mathrm{mol} / \mathrm{l}$ (range, $4-13 \mu \mathrm{mol} / \mathrm{l}$ ) and the LDH was $275 \mathrm{U} / 1$ (range, 268-281 U/l) (Fig. 1). The patterns of laboratory changes in patients with HT are summarized in supplementary Table SII. 


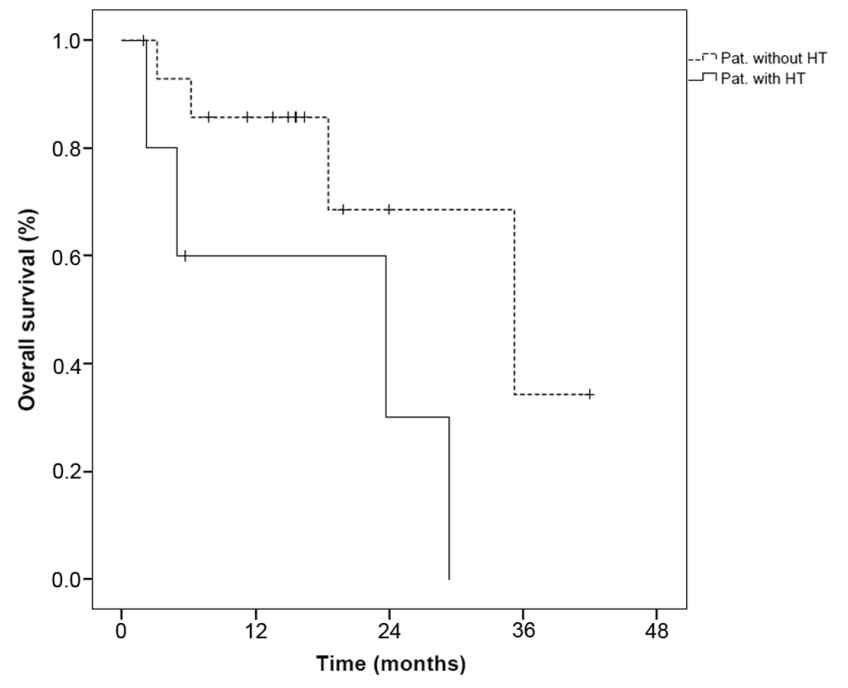

Figure 2. Overall survival of patients with gastrointestinal stromal tumors treated with regorafenib with and without HT. HT, hepatic toxicity.

Survival. The median cPFS under treatment with regorafenib was 8.6 months [95\% confidence interval (CI): 0-17.8 months], while the median OS from initiation of regorafenib treatment was 29.3 months (95\% CI: 17.1-41.5 months). When comparing HT with non-HT patients, no significant differences in cPFS or OS were observed (cPFS log-rank $\mathrm{P}=0.9$; OS log-rank $\mathrm{P}=0.07$; Fig. 2).

\section{Discussion}

Tyrosine kinase inhibitor-induced HT has been previously described, but its relevance in GIST patients receiving regorafenib treatment has not been clearly defined by previous studies (9). While the pivotal phase III trial of regorafenib in advanced GIST initially did not report any severe HT, unlike the phase III trial of regorafenib in colorectal cancer, our data demonstrated that almost one-quarter of patients treated with regorafenib $(5 / 21$; $23.5 \%$ ) exhibited laboratory and/or clinical signs of HT $(2,3)$.

The pivotal phase III trial of regorafenib vs. placebo in patients with refractory GIST did not initially report relevant HT, apart from 1 case of drug-related fatal hepatic failure (2). Only elevation of AST levels was observed in $8 \%$ of the cases in a subsequent publication (12). Similarly, a phase III trial of regorafenib monotherapy for metastatic colorectal cancer only reported hyperbilirubinemia in 65/500 (11\%) of the patients, whereas no other signs of HT were reported (3). The phase III trial of regorafenib monotherapy in patients with hepatocellular carcinoma revealed an increase in bilirubin, ALT and AST as a drug related adverse event. Elevated drug related bilirubin was found in $25 \%$ (95/374) of patients treated with regorafenib, while 1 patient experienced grade 4 toxicity. Drug-related elevation in AST was observed in $18 \%$ of patients treated with regorafenib, while 3 patients experienced grade 4 toxicity (4). HT as predefined toxicity has been most sufficiently reported in two retrospective studies of regorafenib in GIST from the UK. First, a report of 20 patients treated within the UK Managed Access Program revealed hepatobiliary toxicity in 10\% (severe in 5\%) of the patients; more importantly, a recent single-center study of 50 patients reported HT in only $2 \%$ of the patients $(13,14)$. Hence, previous studies of regorafenib in GIST have reported an
HT incidence of only up to $10 \%$, while a review of HT in patients with solid neoplasias treated with tyrosine kinase inhibitors reported elevated AST, ALT or bilirubin levels in 21-47.3\% of the treated patients, ultimately underlining the need for awareness of HT during tyrosine kinase inhibitor treatment (9).

Reasonable explanations for differences in HT between study populations and individuals treated with regorafenib outside the trials remain elusive. Due to the small sample size, retrospective design of the analysis and lack of liver biopsies, a convincing explanation is not possible. Of note, a selection bias is possible, since all patients in the present study have been treated at German tertiary care centers. However, dose reductions of regorafenib in our cohort were necessary in $71.4 \%$ due to any adverse event, which is very similar to the $72.0 \%$ dose reductions in the phase III study of regorafenib in GIST (2). Therefore, differences in dosing and therapeutic management cannot explain the discrepancy in reported HT. Furthermore, a clear definition of HT as an integrative term for an adverse event that may be defined by complex changes in a number of laboratory values is not available, and may explain HT being previously less recognized and/or underreported. Interestingly, hepatic metastases prior to initiation of regorafenib treatment were reported in $85.7 \%$ ( $n=24 / 28$ ) of the patients in an analysis of GIST by Schvartsman et al, which is comparable to our reported incidence of liver metastasis in $90.5 \%$ of regorafenib-treated patients (15). However, whether this associated with the occurrence of HT remains elusive. Furthermore, at least in our cohort, no association with hepatotoxic medication or intrahepatic tumor progression was apparent as a possible etiology for the observed HT. Other possible explanations for HT, such as UGT1A1 polymorphisms, suggested for pazopanib-associated HT, remain speculative (16). Differences in metastatic spreading patterns within the liver, as well as supportive liver treatment or previous liver interventions, may also affect the course of disease. Effect size and multifactorial reasoning do not permit any definitive conclusions on these issues.

In conclusion, taking the present data as well as previous studies of regorafenib treatment in other tumor patients into consideration, it may be suggested that HT in GIST patients treated with regorafenib has been underreported thus far. However, clinical monitoring and adequate therapy management allows continuation of regorafenib treatment. Regorafenib administration may achieve a durable palliative result, while even significant HT, once adequately monitored, may not lead to a compromised outcome in these heavily pretreated patients.

\section{Acknowledgements}

Not applicable.

\section{Funding}

No funding was received.

\section{Availability of data and materials}

Raw data were generated at Hannover Medical School. Derived data supporting the findings of the present study are available from the corresponding author on reasonable request. 


\section{Authors' contributions}

VG, PI, BK, KH and HGK designed the project. $\mathrm{MH}, \mathrm{BK}, \mathrm{KH}$, $\mathrm{HE}$, and HGK were responsible for data collection. PI and $\mathrm{HE}$ performed statistical analysis and wrote the manuscript, with support from AG and VG. All authors discussed the results and contributed to the final manuscript.

\section{Ethics approval and consent to participate}

The present study was performed with approval from the Ethics Committee of Hannover Medical School (no. 3167-2016). No further patient consent was deemed mandatory.

\section{Patient consent for publication}

Written consent is not applicable. Patient data were anonymized.

\section{Competing interests}

PI has an advisory role for Novartis, Bayer and Pfizer and has received travel grants from Novartis and Bayer. HE has received travel grants from Ipsen. BK has an advisory role for Bayer. AG has received advisory board honoraria from Novartis and Celgene. VG has an advisory role for Novartis, Pfizer, GSK and Bayer and has received honoraria for lectures from Novartis, Pfizer and GSK. MH, KH and HGK declare that they have no competing interests to disclose.

\section{References}

1. Wilhelm SM, Dumas J, Adnane L, Lynch M, Carter CA, Schütz G, Thierauch KH and Zopf D: Regorafenib (BAY 73-4506): A new oral multikinase inhibitor of angiogenic, stromal and oncogenic receptor tyrosine kinases with potent preclinical antitumor activity. Int J Cancer 129: 245-255, 2011.

2. Demetri GD, Reichardt P, Kang YK, Blay JY, Rutkowski P, Gelderblom H, Hohenberger P, Leahy M, von Mehren M, Joensuu $\mathrm{H}$, et al: Efficacy and safety of regorafenib for advanced gastrointestinal stromal tumours after failure of imatinib and sunitinib (GRID): An international, multicentre, randomised, placebo-controlled, phase 3 trial. Lancet 381: 295-302, 2013.

3. Grothey A, Van Cutsem E, Sobrero A, Siena S, Falcone A, Ychou M, Humblet Y, Bouché O, Mineur L, Barone C, et al: Regorafenib monotherapy for previously treated metastatic colorectal cancer (CORRECT): An international, multicentre, randomised, placebo-controlled, phase 3 trial. Lancet 381: 303-312, 2013.

4. Bruix J, Qin S, Merle P, Granito A, Huang YH, Bodoky G, Pracht M, Yokosuka O, Rosmorduc O, Breder V, et al: Regorafenib for patients with hepatocellular carcinoma who progressed on sorafenib treatment (RESORCE): A randomised, double-blind, placebo-controlled, phase 3 trial. Lancet 389: 56-66, 2017.
5. Roderburg C, Özdirik B, Wree A, Demir M and Tacke F: Systemic treatment of hepatocellular carcinoma: From sorafenib to combination therapies. Hepat Oncol 7: HEP20, 2020.

6. Sternberg CN, Davis ID, Mardiak J, Szczylik C, Lee E, Wagstaff J, Barrios CH, Salman P, Gladkov OA, Kavina A, et al: Pazopanib in locally advanced or metastatic renal cell carcinoma: Results of a randomized phase III trial. J Clin Oncol 28: 1061-1068, 2010.

7. Kudo M, Imanaka K, Chida N, Nakachi K, Tak WY, Takayama T, Yoon JH, Hori T, Kumada H, Hayashi N, et al: Phase III study of sorafenib after transarterial chemoembolisation in Japanese and Korean patients with unresectable hepatocellular carcinoma. Eur J Cancer 47: 2117-2127, 2011.

8. Motzer RJ, Hutson TE, Tomczak P, Michaelson MD, Bukowski RM, Oudard S, Negrier S, Szczylik C, Pili R, Bjarnason GA, et al: Overall survival and updated results for sunitinib compared with interferon alfa in patients with metastatic renal cell carcinoma. J Clin Oncol 27: 3584-3590, 2009.

9. Iacovelli R, Palazzo A, Procopio G, Santoni M, Trenta P, De Benedetto A, Mezi S and Cortesi E: Incidence and relative risk of hepatic toxicity in patients treated with anti-angiogenic tyrosine kinase inhibitors for malignancy. Br J Clin Pharmacol 77: 929-938, 2014

10. World Medical Association: World medical association declaration of helsinki: Ethical principles for medical research involving human subjects. JAMA 310: 2191-2194, 2013.

11. Cancer Therapy Evaluation Programm D NCI, NIH, DHHS: Common terminology criteria for adverse events v4.0 (CTCAE), 2009.

12. Komatsu Y, Doi T, Sawaki A, Kanda T, Yamada Y, Kuss I, Demetri GD and Nishida T: Regorafenib for advanced gastrointestinal stromal tumors following imatinib and sunitinib treatment: A subgroup analysis evaluating Japanese patients in the phase III GRID trial. Int J Clin Oncol 20: 905-912, 2015.

13. Kollàr A, Maruzzo M, Messiou C, Cartwright E, Miah A, Martin-Liberal J, Thway K, McGrath E, Dunlop A, Khabra K, et al: Regorafenib treatment for advanced, refractory gastrointestinal stromal tumor: A report of the UK managed access program. Clin Sarcoma Res 4: 17, 2014.

14. Chamberlain F, Farag S, Williams-Sharkey C, Collingwood C, Chen L, Mansukhani S, Engelmann B, Al-Muderis O, Chauhan D, Thway K, et al: Toxicity management of regorafenib in patients with gastro-intestinal stromal tumour (GIST) in a tertiary cancer centre. Clin Sarcoma Res 10: 1, 2020.

15. Schvartsman G, Wagner MJ, Amini B, Zobniw CM, Trinh VA, Barbo AG, Lin HY, Wang WL, Conley AP, Ravi V, et al: Treatment patterns, efficacy and toxicity of regorafenib in gastrointestinal stromal tumour patients. Sci Rep 7: 9519, 2017.

16. Xu CF, Reck BH, Xue Z, Huang L, Baker KL, Chen M, Chen EP, Ellens HE, Mooser VE, Cardon LR, et al: Pazopanib-induced hyperbilirubinemia is associated with Gilbert's syndrome UGT1A1 polymorphism. Br J Cancer 102: 1371-1377, 2010. 